\title{
Piezoelectric Elastic Modulus Scanner
}

National Cancer Institute

\section{Source}

National Cancer Institute. Piezoelectric Elastic Modulus Scanner. NCI Thesaurus. Code C126976.

A device that maps the elasticity modulus of a material surface using a piezoresonance probe during scanning microscopy. 